\title{
RSK2 Signaling in Medial Habenula Contributes to Acute Morphine Analgesia
}

\author{
Emmanuel Darcq ${ }^{1,3}$, Katia Befort', Pascale Koebel', Solange Pannetier', Megan K Mahoney',2, \\ Claire Gaveriaux-Ruff', André Hanauer' and Brigitte L Kieffer*, I \\ 'Institut de Génétique et de Biologie Moléculaire et Cellulaire, Centre National de la Recherche Scientifique/lnstitut National de la Santé et de la \\ Recherche Médicale/Université de Strasbourg, Illkirch, France; '2Queen's University, Department of Psychology, Kingston, ON, Canada
}

\begin{abstract}
It has been established that mu opioid receptors activate the ERKI/2 signaling cascade both in vitro and in vivo. The Ser/Thr kinase RSK2 is a direct downstream effector of ERKI/2 and has a role in cellular signaling, cell survival growth, and differentiation; however, its role in biological processes in vivo is less well known. Here we determined whether RSK2 contributes to mu-mediated signaling in vivo. Knockout mice for the rsk2 gene were tested for main morphine effects, including analgesia, tolerance to analgesia, locomotor activation, and sensitization to this effect, as well as morphine withdrawal. The deletion of RSK2 reduced acute morphine analgesia in the tail immersion test, indicating a role for this kinase in mu receptor-mediated nociceptive processing. All other morphine effects and adaptations to chronic morphine were unchanged. Because the mu opioid receptor and RSK2 both show high density in the habenula, we specifically downregulated RSK2 in this brain metastructure using an adeno-associated-virally mediated shRNA approach. Remarkably, morphine analgesia was significantly reduced, as observed in the total knockout animals. Together, these data indicate that RSK2 has a role in nociception, and strongly suggest that a mu opioid receptor-RSK2 signaling mechanism contributes to morphine analgesia at the level of habenula. This study opens novel perspectives for both our understanding of opioid analgesia, and the identification of signaling pathways operating in the habenular complex.

Neuropsychopharmacology (2012) 37, 1288-1296; doi:10.1038/npp.201 I.316; published online 4 January 2012
\end{abstract}

Keywords: opiate; mu opioid receptor; habenular complex; knockout mice; shRNA knockdown; withdrawal

\section{INTRODUCTION}

RSK2 belongs to the ribosomal S6 kinase $90 \mathrm{kDa}$ family (RSK or p90RSK) that includes four members (RSK1 to 4). These serine/threonine kinases are substrates of extracellular-regulated kinases 1 and 2 (ERK1/2) that in turn activate both cytosolic and nuclear targets. Their roles in cellular signaling, cell survival, growth, and differentiation have been well established (Anjum and Blenis, 2008), however their implication in biological processes in vivo is less well known. RSK2 was identified as a disease gene responsible for the Coffin-Lowry syndrome (Temtamy et al, 1975; Trivier et al, 1996; Pereira et al, 2010), an X-linked disease with progressive skeletal abnormalities, facial dimorphism, strong psychomotor impairment, and mental retardation (Pereira et al, 2010). The severe consequences of

*Correspondence: Professor BL Kieffer, IGBMC, Department of Neurobiology and Genetics, Parc d'innovation I rue Laurent Fries BP 10142, 67404 Illkirch cedex, France, Tel: + 33038865 5693, Fax: + 33038865 5604, E-mail: briki@igbmc.fr

${ }^{3}$ Current address: The Ernest Gallo Clinic and Research Center, University of California, San Francisco, Emeryville, CA, USA

Received 27 August 2011; revised 16 November 2011; accepted 17 November 2011
RSK2 deficiency in humans underscore the important role for this member of the RSK family in both peripheral and central physiology.

$\mathrm{Mu}$ opioid receptors are the primary molecular target of both clinically useful and abused opiates in vivo, and have a major role in pain control and drug reward (Kieffer and Gaveriaux-Ruff, 2002; Contet et al, 2004; Le Merrer et al, 2009). These receptors, as for most Gi/o protein-coupled receptors, activate the ERK1/2 signaling cascade. Morphineinduced ERK1/2 phosphorylation was largely demonstrated in cellular models, and also evidenced in vivo following both acute and chronic treatments in rodent models (Eitan et al, 2003; Valjent et al, 2004). Further, ERK1/2 inhibitors modulate responses to morphine, including acquisition (Ozaki et al, 2004), reconsolidation, (Valjent et al, 2006) and reinstatement (Li et al, 2008) of morphine-conditioned place preference, as well as morphine withdrawal (Cao et al, 2005). RSKs are direct downstream effectors of ERK1/2 (Anjum and Blenis, 2008) and may therefore contribute to morphine activity in vivo. This possibility, however, has not been investigated thus far.

RSK2 is one of the most abundant RSK forms in the brain and is expressed at the level of forebrain regions, including cortex and hippocampus, where it is best 
described (Zeniou et al, 2002). We also recently found prominent expression of RSK2 at the level of the habenular complex (Darcq et al, 2011), a brain area that modulates many neural processes and is the subject of increasing attention (Lecourtier and Kelly, 2007; Hikosaka, 2010). Importantly, the habenula is a main site for mu opioid receptor expression, (Mansour et al, 1995; Kitchen et al, 1997) and therefore represents a possible brain substrate for functional interaction between $\mathrm{mu}$ opioid receptor and RKS2 signaling.

Here, we first tested the hypothesis that RSK2 mediates some of the morphine activities in vivo. Using RSK2deficient mice, we investigated morphine analgesia, a main effect of acute morphine, as well as locomotor sensitization and physical dependence representing classical adaptive responses to the drug. Because analgesia is reduced in mutant mice, we further hypothesized that RSK2 is implicated in morphine analgesia at the level of habenular circuitry. Using virally mediated shRNA RSK2 knockdown, we demonstrate for the first time that RSK2 signaling in habenula contributes to morphine analgesia.

\section{MATERIALS AND METHODS}

\section{Animals}

Knockout mrsk2 (mrsk2 KO) mice were generated as described (Yang et al, 2004). Heterozygous females carrying the mutation were crossed with $\mathrm{C} 57 \mathrm{BL} / 6 \mathrm{~J}$ males for at least 20 generations to obtain a $99.99 \%$ C57BL/6J genetic background. Wild-type (WT) and knockout animals used in this study were from the same littermates. Eight-week-old male mice were housed 4 per cage, under standard laboratory conditions ( $12 \mathrm{~h}$ dark/light cycle/light on at 0700 hours). Food and water were available ad libitum. For stereotaxic experiments, wild-type C57BL/6J mice were used (Charles River, St-Germain-sur-l'Arbresle, France). All experimental procedures were conducted according to standard ethical guidelines (European Communities Council Directive of 24 November 1986 (86/609/EEC)).

\section{Drugs}

Morphine hydrochloride (Francopia, Sanofi Synthelabo Laboratories, Paris, France) and Naloxone (Sigma-Aldrich, Lyon, France) were prepared in saline $(0.9 \%$ sodium chloride). For surgery, mice were anaesthetized using ketamine/xylazine $(100 / 10 \mathrm{mg} / \mathrm{kg})$. Drugs were administered subcutaneously (naloxone) or intraperitoneally (morphine and anesthetics) in a volume of $10 \mathrm{ml} / \mathrm{kg}$.

\section{Behavioral Testing}

Morphine-induced analgesia. Mice were administered with 3,5 or $10 \mathrm{mg} / \mathrm{kg}$ morphine or saline and analgesia tested using the tail immersion test as previously described (Scherrer et al, 2004; Contet et al, 2008). Briefly, $45 \mathrm{~min}$ following injection, the tail was immersed in a water bath set at $52^{\circ} \mathrm{C}$ and tail withdrawal latencies were measured with a cut-off time of $10 \mathrm{~s}$. Baseline responding was measured for 3 days before testing and just before the first injection. To test tolerance of morphine analgesia (twice daily, $10 \mathrm{mg} / \mathrm{kg}$ ), latencies were measured once daily for 9 days, $45 \mathrm{~min}$ following the morning injection.

Morphine-induced locomotor sensitization. Locomotor activity was measured in translucent activity boxes $(33 \times 15 \times 14 \mathrm{~cm})$ set on an infrared-emitting floor (IR lighted cages, Viewpoint) under a 40-lux indirect lighting, with horizontal movements monitored using a video tracking device (Videotrack, Viewpoint). Mice were placed in the activity cages for $1 \mathrm{~h}$ (basal exploration), injected with saline $(1 \mathrm{ml} / \mathrm{kg})$, and returned to the cages for $1 \mathrm{~h}$. Mice were then injected with morphine $(40 \mathrm{mg} / \mathrm{kg})$ or saline and activity was recorded for $2 \mathrm{~h}$. Distance traveled every 10-min was recorded over the whole session (ViewPoint, France). For locomotor sensitization, mice were injected twice a week with $40 \mathrm{mg} / \mathrm{kg}$ morphine or saline (ip) and activity was measured on day 1, 4, 8, 11, and 15 (adapted from (Contet et al, 2008)).

Morphine-induced dependence. Mice were injected with escalating doses of morphine $(20,40,60,80,100 \mathrm{mg} / \mathrm{kg})$ or saline, twice daily for 5 days and received a single $100 \mathrm{mg} / \mathrm{kg}$ injection on day 6. To measure physical dependence, withdrawal was precipitated by naloxone $(1 \mathrm{mg} / \mathrm{kg}) 2 \mathrm{~h}$ after the last morphine injection (Contet et al, 2008). Somatic withdrawal signs (jumps, paw and body tremors, activity, shakes, sniffings, teeth chattering, ptosis, and piloerection) were scored per 5 -min period for 20 min under an indirect 50 lux lighting, and a global withdrawal score was calculated as described (Le Merrer et al, 2011).

\section{Construction of RSK2-ShRNA and AAV Purification}

Viral production and shRNA design were carried out using AAV2-helper-free system (Stratagene, France). Two shRNA constructs were used in this study, AAV2-shRSK2 and AAV2-shScramble control, both containing a fluorescent eGFP reporter. In vitro as well as in vivo validation of RSK2 knockdown are described elsewhere (Darcq et al, 2011).

\section{Surgery and Virus Delivery}

With a stereotaxic apparatus (Unimecanique, France), AAV2-shRSK2 and AAV2-shScramble were infused into the medial habenula (Paxinos and Franklin, 2001) under ketamine/xylazine anesthesia. Purified AAV2 were infused bilaterally ( $1.5 \mu \mathrm{l}$ per side/15 min) using a 5 - $\mu$ l microsyringe (SGE Analytical Science, Australia) and the needle was held in place for an additional $10 \mathrm{~min}$ before being removed. Coordinates for the injections were (anteroposterior, $-1.34 \mathrm{~mm}$ from bregma; mediolateral, $\pm 0.3 \mathrm{~mm}$ from midline; dorsoventral, $-3.0 \mathrm{~mm}$ from dura skull surface) (see (Darcq et al, 2011)). Following surgery, mice were single housed for $48 \mathrm{~h}$ and then placed back in their home cage for 6 weeks prior to behavioral testing.

In total, we have prepared two large animal cohorts for behavior (for each cohort $n=20$ shRSK2 mice and $n=20$ shScramble mice). In order to optimize the use of our animals and meet ethical requirements, we used animals in two distinct experimental sequences that involved lithium place conditioning in a first sequence (previously published study, Darcq et al, 2011) and morphine treatments for the 
second sequence (this study). To exclude behavioral interference between the two experimental series, animals were left at rest for 1 week before receiving morphine injections. Most importantly also, a number of animals either did not experience the lithium-conditioning paradigm (entirely naïve), or only received saline in the lithium conditioning experiment (lithium naïve), so that data analysis could be performed on naïve and previously lithium-exposed animals separately, and ultimately pooled (see Results section and Figure $4 \mathrm{~b}$ ). The detailed timeline of the entire experimental sequence and the composition of each group for the two cohorts is shown in Supplementary Figure $1 \mathrm{~A}$ and $\mathrm{B}$.

\section{Tissue Preparation and in situ Hybridization}

Brain sections $(20 \mu \mathrm{m})$ were analyzed for injection accuracy into the habenula using fluorescent microscopy (Darcq et al, 2011) or processed for in situ hybridization using digoxigenin-labeled RNA probes, as previously described (Chotteau-Lelievre et al, 2006), with a 276-bp Rsk2 probe (Zeniou et al, 2002) and a 608-bp Oprm1 probe (accession number: NM_001039652; bp 654-1262).

\section{Statistical Analysis}

Data were analyzed using analysis of variance (ANOVA), computed with the Statistical Program for the Social Sciences (SPSS; V.19). Degrees of freedom for repeated measures were adjusted using the Greenhouse-Geisser correction if assumptions of sphericity were violated. When significant main effects were observed in between-subjects analyses, post-hoc tests were performed using a Bonferroni correction. Within-subjects significant main effects were further analyzed with post-hoc paired $t$-tests with an $\propto$ correction for the number of comparisons made. Significant interactions were analyzed with simple main effects.

\section{RESULTS}

\section{Morphine Analgesia is Reduced in RSK2 Knockout Mice}

We first investigated morphine analgesia in the tail immersion test, a classical pain paradigm used to assess acute analgesic effects of opiates. We compared the analgesic properties of three doses of morphine in wildtype (WT) and mrsk2 knockout (KO) animals (Figure 1a). We used a two-way ANOVA with drug dose and genotype as between-subjects factors. Results revealed significant main effects of both drug dose $(\mathrm{F}(3,111)=80.68, p<0.001)$ and genotype $(\mathrm{F}(1,111)=23.66, p<0.001)$ and a significant dose $\times$ genotype interaction $(\mathrm{F}(3,111)=11.55, p<0.001)$. Simple main effects of the dose $\times$ genotype interaction revealed no genotype differences at $3 \mathrm{mg} / \mathrm{kg}$ morphine. At 5 and $10 \mathrm{mg} / \mathrm{kg}$ morphine doses, a significant increase of latencies was observed in both WT and mrsk2 KO animals (all $p$-values $<0.001$ ). Furthermore, at these doses, KO animals had significantly shorter latencies than WT animals (all $p$-values $<0.01$ ). There was no difference between WT and mrsk2 $\mathrm{KO}$ animals following saline injection. In conclusion, these data show a reduction of morphine analgesia in mrsk2 $\mathrm{KO}$ mice, suggesting that RSK2 signaling
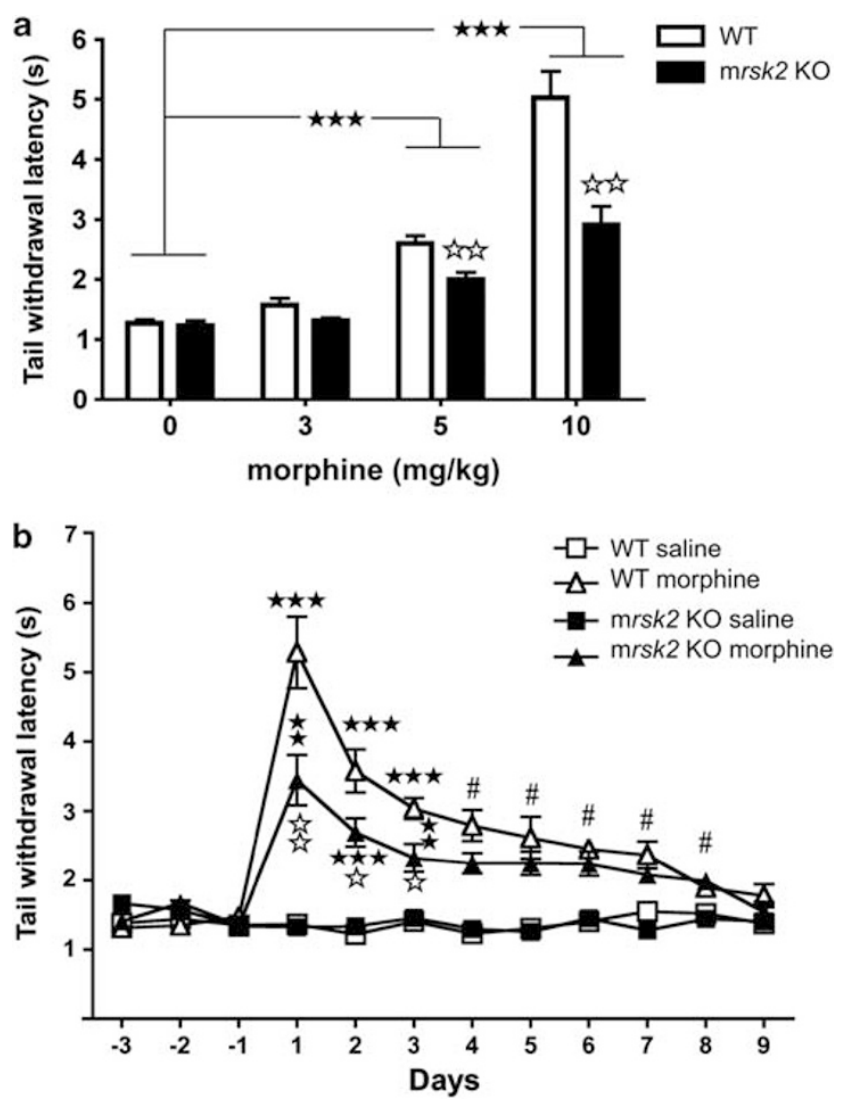

Figure I Decreased acute morphine effect on tail-withdrawal latency in rsk2 knockout mice (mrsk2 KO) mice. (a) Morphine produces analgesia in the tail immersion test on both wild-type (WT) and mrsk2 KO. Tail withdrawal latencies were measured at $52^{\circ} \mathrm{C}$ following morphine injection at $3 \mathrm{mg} / \mathrm{kg}$ (a, WT saline $n=7$, morphine $n=10$; $\mathrm{KO}$ saline $n=6$, morphine $n=5$ ), $5 \mathrm{mg} / \mathrm{kg}$ (b, WT saline $n=9$, morphine $n=16 ; \mathrm{KO}$ saline $n=9$, morphine $n=1 \mathrm{I}$ ) and $10 \mathrm{mg} / \mathrm{kg}$ (c, WT saline $n=9$, morphine $n=14 ; \mathrm{KO}$ saline $n=10$, morphine $n=14)$. Latencies were significantly increased at 5 and $10 \mathrm{mg} / \mathrm{kg}$ in both genotypes. Interestingly, morphine analgesia is significantly reduced in mrsk $2 \mathrm{KO}$ mice at both 5 and $10 \mathrm{mg} / \mathrm{kg}$ compared with WT controls. Values represent the mean \pm SEM. Main effect of drug: $\star \star \star p<0.00$ l. Effect of genotype (comparison between WT and mrsk2 $\mathrm{KO}$ within each treatment condition): $p<0.01$. (b) Tolerance to morphine analgesia develops in WT and mrsk2 $\mathrm{KO}$ mice. Morphine was injected twice daily $(10 \mathrm{mg} / \mathrm{kg})$ and tail withdrawal latencies were measured 45 min following the morning saline or morphine injection (WT saline $n=6$, morphine $n=8$; mrsk2 $\mathrm{KO}$ saline $n=7$, morphine $n=8$ ). As in the previous experiment (a), mrsk2 $\mathrm{KO}$ mice showed reduced morphine sensitivity at days 1-3, however both genotypes showed tolerance with latencies back to saline-injected levels by day 9. Values represent the mean \pm SEM. Main effect of drug: ${ }^{\#} p<0.05$; simple main effects of drug

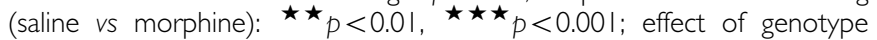
(comparison between WT and mrsk2 $\mathrm{KO}$ within each treatment condition): 施 $p<0.05$, 斿 $p<0.01$.

contributes to morphine analgesia in the tail immersion test.

We also tested whether repeated injections of morphine would lead to analgesic tolerance that would differ between mrsk2 KO and WT mice. We used the tail immersion test as described previously. Tail-withdrawal latency was measured for 3 days prior to testing (habituation), then once daily for 9 days (Figure 1b). Analgesic tolerance was analyzed using a three-way ANOVA with drug and genotype as between-subjects factors and day as a within subjects factor. Significant main effects of day $(\mathrm{F}(8,200)=11.99, p<0.001)$, 
drug $(\mathrm{F}(1,25)=294.49, p<0.001)$, and genotype $(\mathrm{F}(1,25)=$ $19.57 p<0.001)$ were observed. Simple main effects of the significant three-way interaction $(F(8,200)=3.13$, $p=0.031)$ revealed morphine $(10 \mathrm{mg} / \mathrm{kg})$ analgesia for both genotypes on days $1-3$, with reduced latencies for mrsk2 KO compared with WT $(p<0.05)$ (Figure $1 \mathrm{~b})$. We observed a reduction of analgesia with repeated morphine injections; mrsk2 $\mathrm{KO}$ and WT animals had similar latencies between days 4-9 $(p>0.05)$, which were back to saline-injected responding by day 9. In conclusion, these data confirm reduced analgesic effects of morphine in the tail withdrawal response for the mrsk2 $\mathrm{KO}$ mice. Also, tolerance to analgesic effects of morphine has developed similarly in both genotypes leading to full analgesic tolerance at 9 days. This result suggests that RSK2 does not contribute to neuroadaptations leading to tolerance.

\section{Locomotor Activation and Sensitization are Unchanged in RSK2 Knockout Mice}

We then investigated morphine-induced hyperlocomotion, a well-documented effect of morphine. WT and mrsk2 KO mice were administered with either saline or morphine (40 mg/kg) (see methods) and the total distance traveled for $2 \mathrm{~h}$ was measured (Figure 2). This morphine effect was examined using a three-way ANOVA with drug and genotype as between-subjects factors and day as a within subjects factor. Significant main effects of day $(\mathrm{F}(4,128)=16.51, p<0.001)$ and drug $(\mathrm{F}(1,32)=163.49$, $p<0.001)$, as well as a significant day $\times$ drug interaction $(\mathrm{F}(4,128)=18.59, p<0.001)$ were observed. There was no significant genotype main effect, or genotype interactions. Simple main effects of the day $\times$ drug interaction revealed a morphine-induced stimulatory effect on locomotion in all animals at all days (all $p$-values $<0.001$ ). Furthermore, sensitization was observed in all treated animals, with a greater distance traveled on consecutive injections, with the exception of day 15 (all $p$-values $<0.001$ ). In conclusion, deletion of RSK2 did not affect morphine hyperlocomotion nor locomotor sensitization in our conditions.

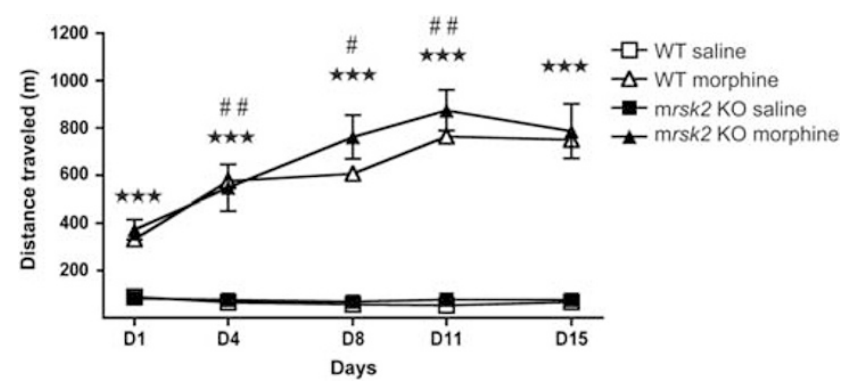

Figure 2 rsk2 gene deletion does not modify morphine-induced locomotor activity. Wild-type (WT) and rsk2 knockout (mrsk2 KO) mice were treated twice a week with either saline or $40 \mathrm{mg} / \mathrm{kg}$ morphine $(n=9 /$ group) to evaluate the development of sensitization to morphine-induced hyperlocomotion. Locomotor activity was monitored for $2 \mathrm{~h}$ following each injection. Values represent the mean \pm SEM of distance traveled during each 2-h session. Main effect of drug: ${ }^{\star \star \star} \star p<0.001$; simple main effect of day (morphine group: comparison made to previous day): ${ }^{*} p<0.05$, \#\# $p<0.01$.

\section{Naloxone-Precipitated Morphine Withdrawal is Maintained in RSK2 Knockout Mice}

We further investigated morphine dependence, a syndrome that engages broad adaptations throughout brain circuits. We induced physical dependence to morphine by repeated injections of ascending doses of morphine $(20-100 \mathrm{mg} / \mathrm{kg})$, twice daily over 6 days. Two hours after the last morphine or saline injection, a single naloxone dose $(1 \mathrm{mg} / \mathrm{kg})$ was administered and withdrawal signs were scored. A global withdrawal score was calculated (adapted from Le Merrer et al, 2011) for WT and mrsk2 KO animals (Figure 3). Twoway ANOVAs examining effects of drug and genotype were performed for each behavior measured. For jumps, main effects of drug $(\mathrm{F}(1,36)=69.67, p<0.001)$, and genotype $(\mathrm{F}(1,36)=16.29, p<0.001)$, and the drug $\times$ genotype interaction $(F(1,36)=5.52, p=0.024)$ were observed. Simple main effects of this interaction revealed that $\mathrm{KO}$ mice jumped significantly more than WT following both saline and morphine injections, indicating that naloxone alone has a measurable effect in $\mathrm{KO}$ animals for this behavior. For teeth chattering, main effects of drug $(\mathrm{F}(1,36)=41.91$, $p<0.001)$, and genotype $(\mathrm{F}(1,36)=5.61, p=0.023)$ were obtained, with increased teeth chattering in mrsk2 KO vs WT. For sniffing, activity, ptosis, and piloerection, main effects of drug were observed $((\mathrm{F}(1,36)=17.19, p<0.001)$, $(\mathrm{F}(1,36)=7.07, p=0.012),(\mathrm{F}(1,36)=74.77, p<0.001)$, and $(\mathrm{F}(1,36)=332.24, \quad p<0.001)$, respectively). No genotype differences were observed for other withdrawal signs (paw and body tremors, shakes). For the global withdrawal score comparison, only the main effect of drug was significant $(\mathrm{F}(1,36)=57.64, p<0.001)$. Overall, morphine-treated mice of both genotypes showed significantly more naloxoneprecipitated withdrawal signs, including increased jumps, sniffing, teeth chattering, ptosis, and piloerection and decreased activity (Figure 3 ). This effect was confirmed by the analysis of the global score, suggesting that RSK2 does not contribute to physical dependence induced by morphine.

\section{Morphine Analgesia is Reduced upon RKS2 Knockdown in the Habenula}

We measured mu opioid receptor (Oprm1) and Rsk2 expression in the adult mouse brain by in situ hybridization. Results of hybridization on adjacent coronal sections show that both transcripts are expressed in habenula, with a high expression in the medial part ( $\mathrm{MHb}$ ) (Figure $4 \mathrm{a})$. In a pilot study, we observed that acute morphine treatment increases the phosphorylated form of ERK in the habenula, suggesting that $\mathrm{mu}$ opioid receptor activation indeed recruits the ERK-RSK2 pathway in this brain structure (Supplementary Figure 2).

We then examined whether RSK2 signaling in the MHb contributes to morphine analgesia. We reduced expression of RSK2 in the $\mathrm{Hb}$ using a virally mediated mrsk2 shRNA knockdown approach, which drastically reduces RSK2 mRNA expression paralleled with a $40 \%$ reduction of protein levels (see our previous report Darcq et al, 2011). Two independent cohorts of 40 animals were injected bilaterally either with adeno-associated virus 2 (AAV2) expressing a mrsk2 shRNA (shRSK2) or a scramble control 

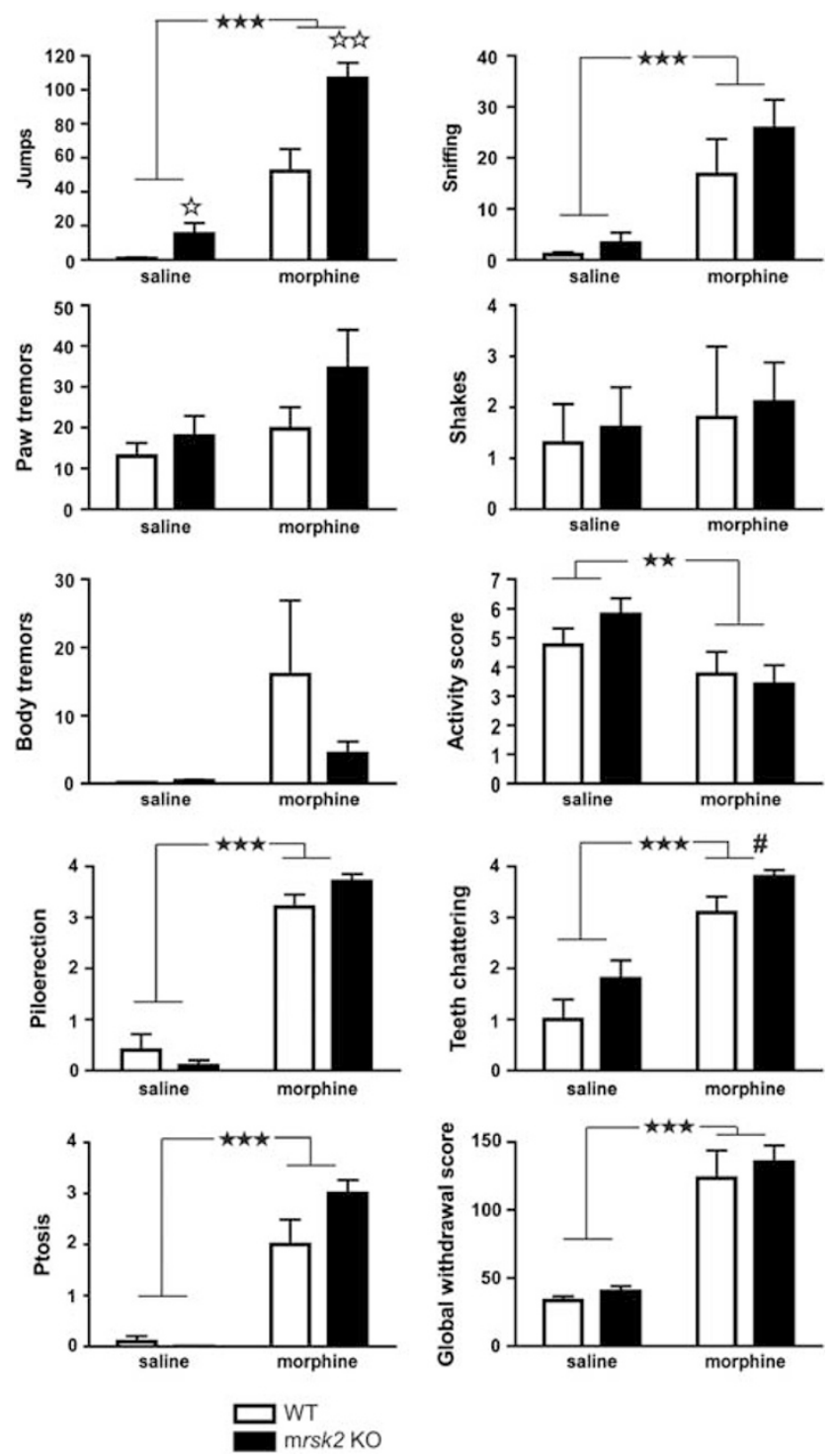

Figure 3 Chronic morphine treatment does not modify withdrawal syndrome in rsk2 knockout mice (mrsk2 KO). Mice were treated twice daily for 5 days with either saline or ascending doses of morphine $(n=10 /$ group/genotype; $20-100 \mathrm{mg} / \mathrm{kg}$ ). On the sixth day, withdrawal syndrome was precipitated by administrating the antagonist naloxone $(I \mathrm{mg} / \mathrm{kg}) 2 \mathrm{~h}$ after the last morphine injection. Somatic signs of withdrawal were recorded for 20 min scored and a global score was calculated (bottom right panel). Overall, morphine-treated mice showed significantly more withdrawal signs than saline-treated mice and no significant difference was observed between mrsk2 KO and wild-type (WT) mice for the global withdrawal score. Values represent the mean \pm SEM. Main effect of drug:

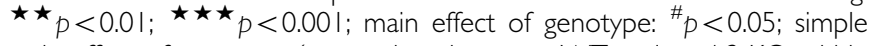
main effect of genotype (comparison between WT and mrsk2 KO within

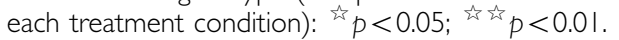

shRNA (shScramble). Treated mice were subjected to behavioral testing, first in a lithium place-conditioning paradigm (6 weeks post injection, data shown in Darcq et al, 2011), then for morphine analgesia using the tail immersion test (8 weeks post injection), and for withdrawal symptoms (9 weeks post injection). This design aimed at optimizing animal usage (see Methods and Supplementary Figure 1A and $\mathrm{B})$. Animals were finally euthanized to control for correct injection site and viral expression (eGFP signal) analysis (Darcq et al, 2011), and altogether, AAV-injected animals (shScramble $n=17$ /group, shRSK2 $n=18$ /group) were tested for morphine responses.

In order to exclude the possibility that prior exposure to lithium-conditioned place aversion influences subsequent morphine analgesia, we analyzed tail withdrawal responses in several distinct subgroups of shScramble- and shRSK2 mice. A first comparison included small subgroups of mice that were not previously tested for lithium conditioning (naïve). Morphine analgesia appeared reduced in shRSK2treated mice (Supplementary Figure 3), and statistical analysis confirmed a significant main effect of morphine $(\mathrm{F}(1,9)=6.106, p<0.05)$ and a main effect of viral treatment $(\mathrm{F}(1,9)=2.889, p=0.1234)$. A second analysis compared groups of mice previously conditioned with lithium (pre-exposed lithium) with mice previously conditioned with saline (pre-exposed saline) or unexposed to the conditioning paradigm (naïve) (Figure 4b). Statistical analysis revealed a significant main effect of morphine $(\mathrm{F}(1,62)=31.862, p<0.001)$ and viral treatment $(\mathrm{F}(1$, $62)=10.785, p<0.002)$ and a significant morphine $\times$ viral treatment interaction $(\mathrm{F}(1,62)=10.824, p<0.002)$. Importantly, there was no main effect of the pretreatment $(\mathrm{F}(1$, $62)=0.278, p=0.5998)$ and no interaction between pretreatment and the main effect of morphine (saline $v \mathrm{~s}$ morphine) $(\mathrm{F}(1,62)=2.311, p=0.5176)$ or between pretreatment and the main effect of viral treatment (shScramble $v s$ shRSK2) $(\mathrm{F}(1,62)=3.820, p=0.4060)$. These results confirm reduced morphine analgesia in shRSK2-treated mice, and indicate that this effect does not differ across preexposed and naïve animals.

Because prior behavioral testing did not modify analgesic responses to morphine, we finally combined data from animals of all the subgroups and used a two-way ANOVA with morphine and viral treatment as between-subjects factors (Figure 4c). Analyses revealed a significant main effect of both morphine $(\mathrm{F}(1,66)=32.75, p<0.001)$ and viral treatment $(\mathrm{F}(1,66)=10.16, p<0.002)$ and a significant morphine $\times$ drug treatment interaction $(\mathrm{F}(1,66)=10.29$, $p<0.002)$. Morphine significantly increased latency in both AAV-injected groups compared with saline groups (all $p$-values <0.001). Furthermore, there was no difference following saline injection between shScramble- and shRSK2-injected animals; however, for morphine-treated mice, shRSK2 animals had significantly shorter latencies than control shScramble animals $(p<0.01)$. Morphine analgesia in the tail response is therefore significantly reduced upon local RSK2 knockdown in the habenula. Remarkably, our data also show that total RSK2 gene knockout (Figure 1) and local RSK2 knockdown both reduce morphine analgesia to similar extent. RSK2 expression at this particular brain site, therefore, largely contributes to morphine analgesia in the tail immersion test.

To address the specificity of RSK2 knockdown in habenula on the analgesic response to morphine, we also investigated the consequences of AAV2-shRSK2 injection on naloxone-precipitated morphine withdrawal. Somatic signs of naloxone-induced morphine withdrawal were measured, as for the knockout study, on mice either injected with shScramble ( $n=17 /$ group) or with shRSK2 ( $n=18$ /group) (Figure $4 \mathrm{~d}$ and Supplementary Figure 4$)$. 
Two-way ANOVAs for effects of drug and virus showed significant drug main effects for the following behaviors: jump $(\mathrm{F}(1,65)=315.24, p<0.001))$, sniffing $(\mathrm{F}(1,65)=45.30, p<0.001))$, activity $(\mathrm{F}(1,65)=177.82$, $p<0.001))$, paw tremors $(\mathrm{F}(1,65)=29.76, p<0.001)$, teeth chattering $((\mathrm{F}(1,65)=823.61, p<0.001))$, ptosis $(\mathrm{F}(1,65)=964.86, p<0.001)$, and piloerection $(\mathrm{F}(1,65)=$ $705.60, p<0.001))$. For teeth chattering, simple main effects of the significant drug $\times$ virus interaction $(\mathrm{F}(1,65)=5.12$, $p=0.027)$ revealed that AAV-shRSK2-injected mice teeth chattered significantly more than shScramble following morphine, but not saline. No significant effects were observed for body tremors or shakes. A significant drug main effect was observed for the global withdrawal score

a
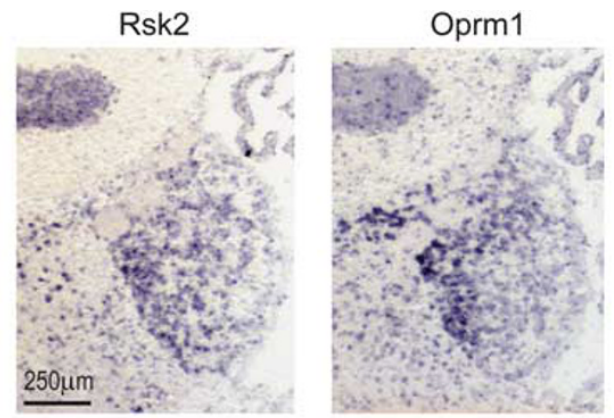

b

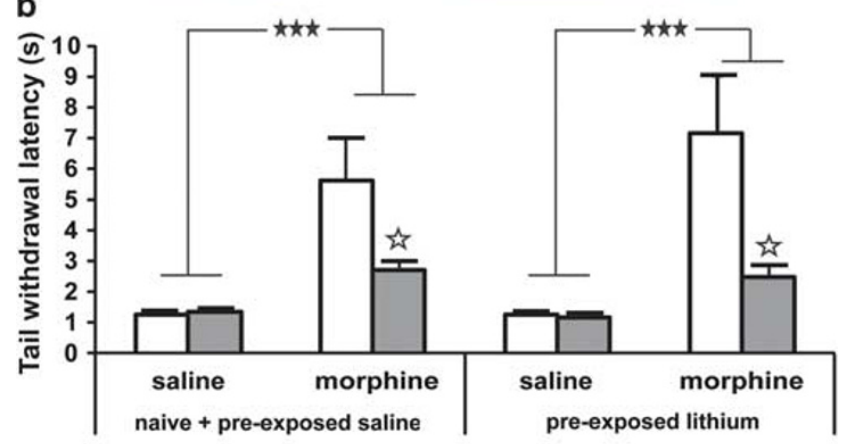

C
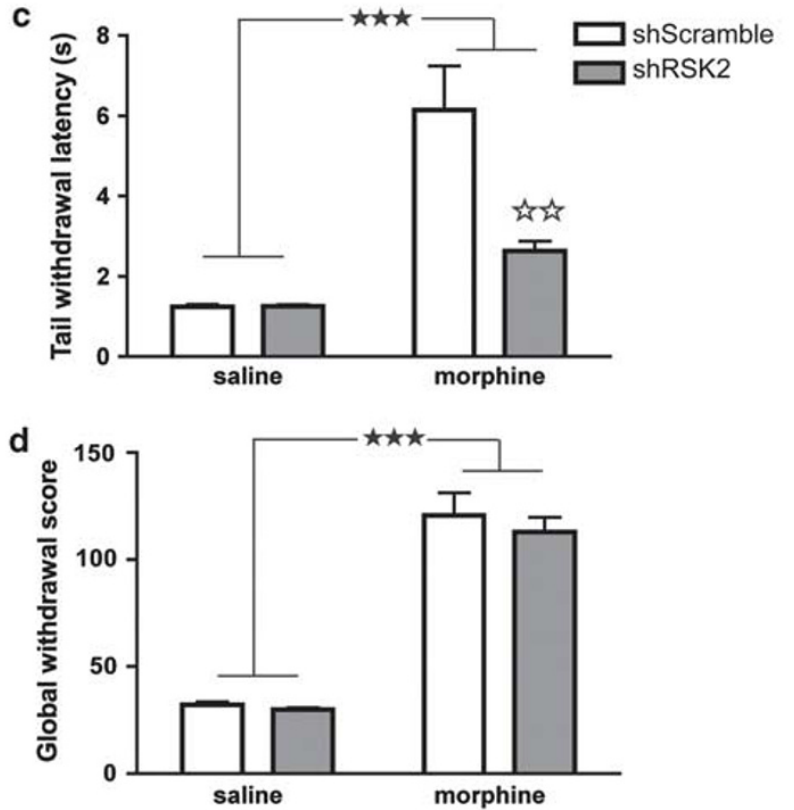

$(\mathrm{F}(1,65)=180.29, p<0.001))$ but no significant effect of virus $(\mathrm{F}(1,65)=0.62, p=0.44))$ and no drug $\times$ virus interaction $(\mathrm{F}(1,65)=0.19, p=0.66)$. Overall, shScrambleand shRSK2-injected mice showed somatic signs of withdrawal. There was no significant difference between morphine-treated mice injected with either virus. This result demonstrates that full expression of RSK2 in MHb is not necessary for the emergence of morphine-withdrawal signs. RSK2 signaling in habenula did not modulate naloxone response. This morphine effect therefore was unchanged in both total and local RSK2-deficient animals.

\section{DISCUSSION}

Our study shows that RSK2 signaling contributes to morphine analgesia in the tail withdrawal response. Furthermore, our finding that similar reductions of morphine analgesia are found following both constitutive mrsk2 gene knockout and local RSK2 mRNA knockdown in the habenula demonstrates that RSK2 operating at the level of habenula neurons contributes to this morphine effect.

In vivo RSK2 has a role in both developmental and cognitive processes, evidenced by the severe phenotype induced by RSK2 deficiency in Coffin-Lowry patients (Hanauer and Young, 2002; Pereira et al, 2010). mrsk2 KO mice recapitulate some of the human symptoms, including peripheral deficits in glycogen metabolism of skeletal muscle (Dufresne et al, 2001) and lipodystrophy (ElHaschimi et al, 2003), as well as central effects on cognition (Poirier et al, 2007). The latter includes impairments of spatial working memory, delayed acquisition of spatial reference memory and long-term spatial memory. In addition, disinhibition and difficulties adapting to a new environment, observed in mutant mice, likely contribute to the cognitive deficits (Poirier et al, 2007). Our study examines major responses to opiates, including analgesia,

Figure 4 Adeno-associated viral (AAV)-mediated expression of RSK2 short hairpin RNA (shRSK2) in the habenula decreases morphine analgesia. (a) Representative images showing the overlapping expression pattern of rsk2 and Oprml (mu opioid receptor) mRNA measured by dig-labeled RNA in situ hybridization on adjacent $20 \mu \mathrm{m}$ coronal brain sections reveals that both transcripts are expressed in medial habenula. (b and c) Effect of RSK2 knockdown on morphine analgesia. Eight weeks following AAV injection, mice were tested for morphine analgesia $(10 \mathrm{mg} / \mathrm{kg})$ in the tail immersion test. (b) Comparison of animals with or without prior behavioral testing (Darcq et al, 20l I) indicates that prior lithium exposure does not influence morphine analgesia. Tail withdrawal responses are shown in animal subgroups either unexposed to lithium (naïve or pre-exposed to saline, ShScramble saline $n=8$, ShScramble morphine $n=11$, ShRSK2 saline $n=10$ and ShRSK2 morphine $n=12$ ) or pre-exposed to lithium (ShScramble saline $n=9$, ShScramble morphine $n=6$, ShRSK2 saline $n=8$ and ShRSK2 morphine $n=6$ ). (c) Combined tail withdrawal data from all subgroups (AAV2-shRSK2, $n=$ I8/group; AAV2-shScramble, $n=17$ | group). Morphine increases tail withdrawal latencies in the two groups, but with significantly lower efficacy in shRSK2 mice. (d) Naloxone-precipitated morphine withdrawal syndrome was observed in the same animals. Global withdrawal score is increased in all morphine-treated mice with no significant differences between shScramble and shRSK2 animals. Values represent the mean \pm SEM. Main effect of morphine: $\star \star \star \star p<0.001$; simple main effect of viral treatment (morphine groups: shScramble vs shRSK2): $p<0.05$ and 2 施 $p<0.01$. 
locomotor activation and physical dependence, and therefore addresses a distinct set of behavioral responses. Our finding of reduced morphine analgesia in mrsk2 knockout mice uncovers a novel role for this kinase in pain control. Whether RSK2 contributes to other forms of sensory processes remains open. In a previous study, mrsk2 knockout mice also showed cortical hyperdopaminergia, suggesting a role of RSK2 in the modulation of dopaminergic neurotransmission (Marques Pereira et al, 2008). Our data further indicate that RSK2 also contributes to opioid neurotransmission, thereby identifying another RSK2-regulated neuromodulatory system.

RSK2 is mainly expressed in the forebrain, at the level of cortex and hippocampus, which contributes partly to higher-order processing of painful stimuli (Basbaum et al, 2009). Interestingly, RSK2 is also highly expressed in medial habenula ((Darcq et al, 2011) and our present results), a highly conserved epithalamic structure. This small region of the brain operates as a metastructure linking extrapyramidal and limbic systems in a bidirectional manner, and integrates cognitive with emotional and sensory processes (Lecourtier and Kelly, 2007; Hikosaka, 2010). The strong RSK2 expression in the habenula suggests that the kinase contributes to a number of habenula-mediated behavioral responses. Further, habenular lesions in the rat produced effects comparable to the deficits observed in m rsk2 KO mice (Thornton and Evans, 1984; Lecourtier and Kelly, 2007; Poirier et al, 2007; Darcq et al, 2011). Our finding that local RSK2 knockdown in the habenula reduces morphine analgesia, together with data from habenular lesions, highlight the important role of RSK2 in regulating morphine responses in the habenula.

There is evidence that habenular neurons contribute to pain control. Nociceptive processing involves a complex circuitry. Namely, this circuitry includes the ascending pain pathways that originate at the level of first-order primary afferent neurons and extend to higher-order thalamic and cortical levels where pain messages are integrated, as well as descending inhibitory pain pathways, which recruit mainly brain stem and spinal cord networks (Fields, 2004; Dickenson and Kieffer, 2006). Previous work has shown that electrical stimulation of the habenula produces analgesia (Cohen and Melzack, 1985, 1986; Terenzi et al, 1990; Terenzi and Prado, 1990), indicating that antinociceptive activity potentially originates from this epithalamic nucleus. This effect was reversed by naloxone (Mahieux and Benabid, 1987), implicating opioid receptor-mediated mechanisms, which are in agreement with the high mu opioid receptor density found in this structure (Kitchen et al, 1997; Le Merrer et al, 2009 and our results). Recent studies, combining analgesic measures following local application of morphine in the habenula and retrograde tracing, suggest that mu opioid receptors in the habenula activate descending antinociceptive pathways (Narita et al, 2008) via dual modulation of excitatory synaptic transmission in $\mathrm{Hb}$ neurons (Hashimoto et al, 2009). Our observations that RSK2 and mu receptors are co-expressed in habenula, and that RSK2 knockdown in the habenula reduces morphine analgesia, provide a molecular mechanism underlying habenular antinociceptive activity. We therefore propose that a mu opioid receptor-RSK2 signaling pathway in $\mathrm{Hb}$ neurons produces analgesia.
To our knowledge, there is no report for a molecular pathway involving mu opioid receptors and RSKs in vivo, as of yet. Opioid receptors are Go/Gi coupled receptors, and their best-described effectors in vivo are voltage-dependent $\mathrm{Ca}+$ channels and inwardly rectifying potassium channels whose activation leads to decreased transmitter release or neuron hyperpolarization, respectively (Williams et al, 2001). The MAPK pathway was reported as another in vivo effector system for opioid receptors. Evidence is mainly based on phosphorylation data (Eitan et al, 2003; Valjent et al, 2004) and in vivo manipulation studies targeting ERK1/2, which is one of the most studied actors of this pathway. ERK1/2 inhibition, following intracerebroventricular administration of a ERK $1 / 2$ phosphorylation blocker or antisense oligonucleotide, decreased morphine withdrawal in the rat (Cao et al, 2005). Conditioned place preference to morphine was abolished when ERK1/2 inhibitors, but not p38 inhibitors, were injected into the VTA (Ozaki et al, 2004), and ERK inhibition during reexposure to the context abolished a previously learned place preference (Valjent et al, 2006). Finally, inhibition of ERK activity in the central amygdala reduced reinstatement of morphine place preference after 14 days of abstinence (Li et al, 2008). Thus, activation of ERK $1 / 2$ is essential for a number of behavioral responses to morphine, and this signaling pathway operates at several brain sites. Studies in cellular models have shown that mitogen and stressactivated protein kinases (MSKs) and RSKs represent two major ERK1/2 substrates (Anjum and Blenis, 2008), and may therefore potentially act as ERK substrates in the brain. In vivo data have established the importance of ERK/MSK signaling in cocaine-induced chromatin remodeling and gene regulation, at the level of striatal neurons (BramiCherrier et al, 2009). Here we show that RSK2, belonging to the other major ERK1/2 substrate family, acts as a downstream effector of mu opioid receptors in the habenula. It is therefore likely that the entire MAPK pathway, including ERK1/2 and RSK2, operates within habenular neurons. Future studies will establish whether kinases of this pathway are activated in vivo at the level of habenula, and potentially identify cytoplasmic or nuclear downstream RSK2 targets (Brami-Cherrier et al, 2009) involved in the modulation of habenular function.

Our data show that RSK2 signaling contributes to morphine analgesia in the tail immersion test. RSK2 also seems to contribute to some naloxone effects, involving an endogenous opioid tone but this effect, which does not involve habenular neurons, requires further investigation. Our data, however, provide no evidence for an implication of RSK2 in any other morphine response that we have tested, including locomotor effects of morphine and physical dependence to morphine. We cannot exclude the possibility that RSK2 is also involved, at least partially, in these effects of morphine, but this contribution was undetectable under our experimental conditions. Locomotor responses and sensitization mostly involve neural activity and adaptations within striatal circuits (Grillner et al, 2005), and drug withdrawal engages broad neural networks throughout the brain. Therefore, it is likely that mu opioid receptors do not, or only weakly, recruit RSK2 signaling for these behavioral responses, either because RSK2 is not expressed in recruited neurons or because the 
$\mathrm{mu}$ receptor induces these behavioral responses via other signaling effectors.

In conclusion, our study shows that RSK2 mediates a specific $\mathrm{mu}$ opioid receptor-mediated response, which operates at the level of the habenula in the brain. These results have two important implications. First, the data reveal that beyond cognition, RSK2 has a role in nociceptive processing. Second, our findings identify a novel mu opioid receptor-mediated signaling mechanism contributing to morphine analgesia in the habenula. This study unfolds novel perspectives for both our understanding of molecular mechanisms involved in opioid analgesia at circuitry levels, and the identification of in vivo signaling pathways operating in the habenula.

\section{ACKNOWLEDGEMENTS}

We thank $O$ Gardon for cloning and supplying the oprm1 probe, J-M Garnier for AAV2-shRNA construction, A-S Kirstetter for technical assistance with stereotaxic experiments, JA Becker for helpful comments and advice. We thank G Duval and D Memedov for animal care. This work was supported by the Centre National de la Recherche Scientifique, Institut National de la Santé et de la Recherche Médicale, and Université de Strasbourg. We thank the European Union (GENADDICT/FP6 005166), and the National Institutes of Health (NIAAA AA-16658 and NIDA DA-16768), for financial support.

\section{DISCLOSURE}

The authors declare no conflict of interest.

\section{REFERENCES}

Anjum R, Blenis J (2008). The RSK family of kinases: emerging roles in cellular signalling. Nat Rev Mol Cell Biol 9: 747-758.

Basbaum AI, Bautista DM, Scherrer G, Julius D (2009). Cellular and molecular mechanisms of pain. Cell 139: 267-284.

Brami-Cherrier K, Roze E, Girault JA, Betuing S, Caboche J (2009). Role of the ERK/MSK1 signalling pathway in chromatin remodelling and brain responses to drugs of abuse. J Neurochem 108: 1323-1335.

Cao JL, He JH, Ding HL, Zeng YM (2005). Activation of the spinal ERK signaling pathway contributes naloxone-precipitated withdrawal in morphine-dependent rats. Pain 118: 336-349.

Chotteau-Lelievre A, Dolle P, Gofflot F (2006). Expression analysis of murine genes using in situ hybridization with radioactive and nonradioactively labeled RNA probes. Methods Mol Biol 326: 61-87.

Cohen SR, Melzack R (1985). Morphine injected into the habenula and dorsal posteromedial thalamus produces analgesia in the formalin test. Brain Res 359: 131-139.

Cohen SR, Melzack R (1986). Habenular stimulation produces analgesia in the formalin test. Neurosci Lett 70: 165-169.

Contet C, Filliol D, Matifas A, Kieffer BL (2008). Morphine-induced analgesic tolerance, locomotor sensitization and physical dependence do not require modification of mu opioid receptor, cdk5 and adenylate cyclase activity. Neuropharmacology 54: 475-486.

Contet C, Kieffer BL, Befort K (2004). Mu opioid receptor: a gateway to drug addiction. Curr Opin Neurobiol 14: 370-378.
Darcq E, Koebel P, Del Boca C, Pannetier S, Kirstetter AS, Garnier JM et al (2011). RSK2 signaling in brain habenula contributes to place aversion learning. Learn Mem 18: 574-578.

Dickenson AH, Kieffer B (2006). Opiates: Basic Mechanisms. Elsevier: London Churchill Livingstone: NY.

Dufresne SD, Bjorbaek C, El-Haschimi K, Zhao Y, Aschenbach WG, Moller DE et al (2001). Altered extracellular signalregulated kinase signaling and glycogen metabolism in skeletal muscle from p90 ribosomal S6 kinase 2 knockout mice. Mol Cell Biol 21: 81-87.

Eitan S, Bryant CD, Saliminejad N, Yang YC, Vojdani E, Keith Jr D et al (2003). Brain region-specific mechanisms for acute morphine-induced mitogen-activated protein kinase modulation and distinct patterns of activation during analgesic tolerance and locomotor sensitization. J Neurosci 23: 8360-8369.

El-Haschimi K, Dufresne SD, Hirshman MF, Flier JS, Goodyear LJ, Bjorbaek C (2003). Insulin resistance and lipodystrophy in mice lacking ribosomal S6 kinase 2. Diabetes 52: 1340-1346.

Fields H (2004). State-dependent opioid control of pain. Nat Rev Neurosci 5: 565-575.

Grillner S, Hellgren J, Menard A, Saitoh K, Wikstrom MA (2005). Mechanisms for selection of basic motor programs - roles for the striatum and pallidum. Trends Neurosci 28: 364-370.

Hanauer A, Young ID (2002). Coffin-Lowry syndrome: clinical and molecular features. J Med Genet 39: 705-713.

Hashimoto K, Amano T, Sakai N, Suzuki T, Narita M (2009). Celldependent physiological synaptic action of morphine in the rat habenular nucleus: morphine both inhibits and facilitates excitatory synaptic transmission. Neurosci Lett 451: 270-273.

Hikosaka O (2010). The habenula: from stress evasion to valuebased decision-making. Nat Rev Neurosci 11: 503-513.

Kieffer BL, Gaveriaux-Ruff C (2002). Exploring the opioid system by gene knockout. Prog Neurobiol 66: 285-306.

Kitchen I, Slowe SJ, Matthes HW, Kieffer B (1997). Quantitative autoradiographic mapping of mu-, delta- and kappa-opioid receptors in knockout mice lacking the mu-opioid receptor gene. Brain Res 778: 73-88.

Le Merrer J, Becker JA, Befort K, Kieffer BL (2009). Reward processing by the opioid system in the brain. Physiol Rev 89: 1379-1412.

Le Merrer J, Befort K, Gardon O, Filliol D, Darcq E, Dembele D et al (2011). Protracted abstinence from drugs of abuse shows common gene network regulations. Addiction Biology (28 September 2011, doi:10.1111/j.1369-1600.2011.00365.x (e-pub ahead of print)).

Lecourtier L, Kelly PH (2007). A conductor hidden in the orchestra? Role of the habenular complex in monoamine transmission and cognition. Neurosci Biobehav Rev 31: 658-672.

Li YQ, Li FQ, Wang XY, Wu P, Zhao M, Xu CM et al (2008). Central amygdala extracellular signal-regulated kinase signaling pathway is critical to incubation of opiate craving. J Neurosci 28: 13248-13257.

Mahieux G, Benabid AL (1987). Naloxone-reversible analgesia induced by electrical stimulation of the habenula in the rat. Brain Res 406: 118-129.

Mansour A, Fox CA, Akil H, Watson SJ (1995). Opioid-receptor mRNA expression in the rat CNS: anatomical and functional implications. Trends Neurosci 18: 22-29.

Marques Pereira P, Gruss M, Braun K, Foos N, Pannetier S, Hanauer A (2008). Dopaminergic system dysregulation in the mrsk2_KO mouse, an animal model of the Coffin-Lowry syndrome. J Neurochem 107: 1325-1334.

Narita M, Hashimoto K, Amano T, Narita M, Niikura K, Nakamura A et al (2008). Post-synaptic action of morphine on glutamatergic neuronal transmission related to the descending antinociceptive pathway in the rat thalamus. $J$ Neurochem 104: 469-478.

Ozaki S, Narita M, Narita M, Ozaki M, Khotib J, Suzuki T (2004). Role of extracellular signal-regulated kinase in the ventral 
tegmental area in the suppression of the morphine-induced rewarding effect in mice with sciatic nerve ligation. J Neurochem 88: 1389-1397.

Paxinos G, Franklin K (2001). The Mouse Brain in Stereotaxic Coordinates. Academic Press: San Diego.

Pereira PM, Schneider A, Pannetier S, Heron D, Hanauer A (2010). Coffin-Lowry syndrome. Eur J Hum Genet 18: 627-633.

Poirier R, Jacquot S, Vaillend C, Soutthiphong AA, Libbey M, Davis $S$ et al (2007). Deletion of the Coffin-Lowry syndrome gene Rsk2 in mice is associated with impaired spatial learning and reduced control of exploratory behavior. Behav Genet 37: 31-50.

Scherrer G, Befort K, Contet C, Becker J, Matifas A, Kieffer BL (2004). The delta agonists DPDPE and deltorphin II recruit predominantly $\mathrm{mu}$ receptors to produce thermal analgesia: a parallel study of mu, delta and combinatorial opioid receptor knockout mice. Eur J Neurosci 19: 2239-2248.

Temtamy SA, Miller JD, Hussels-Maumenee I (1975). The CoffinLowry syndrome: an inherited faciodigital mental retardation syndrome. J Pediatr 86: 724-731.

Terenzi MG, Guimaraes FS, Prado WA (1990). Antinociception induced by stimulation of the habenular complex of the rat. Brain Res 524: 213-218.

Terenzi MG, Prado WA (1990). Antinociception elicited by electrical or chemical stimulation of the rat habenular complex and its sensitivity to systemic antagonists. Brain Res 535: 18-24.
Thornton EW, Evans JA (1984). The effects of lesions of the habenula nucleus on lever press behaviour during a tandem operant schedule with contrasting response requirements. Behav Brain Res 12: 327-334.

Trivier E, De Cesare D, Jacquot S, Pannetier S, Zackai E, Young I et al (1996). Mutations in the kinase Rsk-2 associated with Coffin-Lowry syndrome. Nature 384: 567-570.

Valjent E, Corvol JC, Trzaskos JM, Girault JA, Herve D (2006). Role of the ERK pathway in psychostimulant-induced locomotor sensitization. BMC Neurosci 7: 20.

Valjent E, Pages C, Herve D, Girault JA, Caboche J (2004). Addictive and non-addictive drugs induce distinct and specific patterns of ERK activation in mouse brain. Eur J Neurosci 19: 1826-1836.

Williams JT, Christie MJ, Manzoni O (2001). Cellular and synaptic adaptations mediating opioid dependence. Physiol Rev 81: 299-343.

Yang X, Matsuda K, Bialek P, Jacquot S, Masuoka HC, Schinke T et al (2004). ATF4 is a substrate of RSK2 and an essential regulator of osteoblast biology; implication for Coffin-Lowry Syndrome. Cell 117: 387-398.

Zeniou M, Ding T, Trivier E, Hanauer A (2002). Expression analysis of RSK gene family members: the RSK2 gene, mutated in Coffin-Lowry syndrome, is prominently expressed in brain structures essential for cognitive function and learning. Hum Mol Genet 11: 2929-2940.

Supplementary Information accompanies the paper on the Neuropsychopharmacology website (http://www.nature.com/npp) 